\title{
Def defines a conserved nucleolar pathway that leads p53 to proteasome-independent degradation
}

\author{
Ting Tao ${ }^{1, *}$, Hui Shi ${ }^{1, *}$, Yihong Guan ${ }^{1}$, Delai Huang ${ }^{1}$, Ye Chen ${ }^{1}$, David P Lane ${ }^{3}$, Jun Chen $^{2}$, Jinrong Peng ${ }^{1}$ \\ ${ }^{1}$ Key Laboratory for Molecular Animal Nutrition, College of Animal Sciences, Ministry of Education, Zhejiang University, 866 Yu \\ Hang Tang Road, Hangzhou, Zhejiang 310058, China; ${ }^{2}$ College of Life Sciences, Zhejiang University, 866 Yu Hang Tang Road, \\ Hangzhou, Zhejiang 310058, China; ${ }^{3}$ p53 Laboratory, Agency for Science and Technology Research, 8A Biomedical Grove, \#6-06, \\ Immunos, Singapore 138648
}

p53 protein turnover through the ubiquitination pathway is a vital mechanism in the regulation of its transcriptional activity; however, little is known about p53 turnover through proteasome-independent pathway(s). The digestive organ expansion factor (Def) protein is essential for the development of digestive organs. In zebrafish, loss of function of def selectively upregulates the expression of p53 response genes, which raises a question as to what is the relationship between Def and p53. We report here that Def is a nucleolar protein and that loss of function of $d e f$ leads to the upregulation of p53 protein, which surprisingly accumulates in the nucleoli. Our extensive studies have demonstrated that Def can mediate the degradation of p53 protein and that this process is independent of the proteasome pathway, but dependent on the activity of Calpain3, a cysteine protease. Our findings define a novel nucleolar pathway that regulates the turnover function of p53, which will advance our understanding of p53's role in organogenesis and tumorigenesis.

Keywords: Def; p53; $\Delta 133$ p53/4113p53; ubquitination; Calpain; digestive organ development; nucleolus Cell Research (2013) 23:620-634. doi:10.1038/cr.2013.16; published online 29 January 2013

\section{Introduction}

p53 is under exquisitely fine regulation and acts as a transcription factor that regulates the expression of thousands of genes that control apoptosis, cell cycle arrest, senescence, metabolism, fertility, aging and autophagy [1]. Regulation of p53 turnover is essential to keep p53 activity under control in a normal cell. Multiple E3 ligases that mediate p53 degradation through the ubiquitination-26S proteasome pathway have been identified, including the key E3 ligase $\mathrm{Mdm} 2$ and others such as Pirh2, COP1, Topors, Arf-BP1, Synoviolin, CARP1/2, E6-AP and Trim24 (reviewed in [2]). p53 can also be degraded through the MG132-inhibitable but ubiquiti-

\footnotetext{
*These two authors contributed equally to this work.

Correspondence: Jinrong Peng ${ }^{\mathrm{a}}$, Jun Chen ${ }^{\mathrm{b}}$, David P Lane

${ }^{a}$ E-mail: pengjr@zju.edu.cn

${ }^{b}$ E-mail: chenjun2009@zju.edu.cn

'E-mail: dplane@p53Lab.a-star.edu.sg

Received 24 October 2012; revised 22 November 2012; accepted 28 November 2012; published online 29 January 2013
}

nation-independent pathway [3]. However, very little is known to date about p53 turnover through proteasomeindependent pathway(s), although some reports have shown that certain cytoplasmically abundant calpains might contribute to the degradation of $\mathrm{p} 53$ [4-7].

The nucleolus is also involved in regulating the function of $\mathrm{p} 53$. Disruption of the nucleolus in a cell in response to either internal or external stress normally activates the p53 pathway. In most cases, the activation of p53 is due to the binding of Mdm2 by factors such as ARF [8], PML [9], RPL5 [10] or RPL11 [11], which results in physical separation of Mdm2 from $\mathrm{p} 53$, thus preventing $\mathrm{p} 53$ from degradation by the ubiquitination pathway [12].

Def was first identified as a pan-endodermal-enriched factor that is essential for the growth of digestive organs in zebrafish [13]. Def belongs to a novel protein family that is evolutionally conserved from yeasts to humans and is a component of the ribosomal small subunit (SSU) processome in the nucleolus [14-16]. Human $\Delta 133$ p53 and its zebrafish $\Delta 113 \mathrm{p} 53$ counterpart are N-terminally truncated p53 isoforms whose transcriptional expression 
is initiated by an alternative $\mathrm{p} 53$ promoter that is totally p53 dependent $[13,17-19]$. Both $\Delta 133 p 53$ and $\Delta 113 p 53$ have been found to modulate p53 activity $[17,19]$ and $\Delta 133 p 53$ is upregulated in certain human cancers $[18$, $20,21]$. In the zebrafish def null mutant $\left(\operatorname{def}^{h i 429}\right)$, the transcriptional expression of $\mathrm{p} 53$ response genes, including $4113 p 53, m d m 2$ and $p 21$, is selectively upregulated. However, the level of full-length $p 53$ transcripts was not obviously altered in the $d_{e} f^{h i 429}$ mutant [13], which raises the question of whether $\mathrm{p} 53$ protein is stabilized or overactivated to upregulate the expression of $4113 p 53$ in the def ${ }^{\text {hit29}}$ mutant.

In this report, we analyzed the effect of Def on p53 in both zebrafish and human cells and found that Def triggers the degradation of $\mathrm{p} 53$ and its isoform $\Delta 133 \mathrm{p} 53 /$ $\Delta 113 \mathrm{p} 53$. More importantly, Def-mediated degradation of p53 is dependent on the activity of a specific cysteine proteinase, Calpain 3 (CAPN3), rather than acting through the $26 \mathrm{~S}$ proteasome pathway. Our results demonstrated that both zebrafish and humans share a conserved common nucleolar pathway that mediates p53 degradation.

\section{Results}

Both zebrafish and human Def are localized in the nucleolus

Def homologues in yeast (Upt25p) [14, 15] and Arabidopsis (NOF1) [16] are nucleolar proteins. Zebrafish Def contains a putative nucleolar localization signal (NoLS) [22] (Supplementary information, Figure S1A). Coimmunostaining of Def and the nucleolar marker, Fibrillarin (Fib) [23] showed that Def was colocalized with Fib in the nucleoli in the intestinal epithelia of the wild-type fish at 3.5 days post-fertilization (dpf) but not in those of the $d e f^{\text {hit29 }}$ mutant (Supplementary information, Figure $\mathrm{S} 1 \mathrm{~B}$ and S1C). The human def gene (hu-def) is located in 1q32.2 and is called clorf107. We found that hu-Def was also localized in the nucleolus in MCF-7 cells (Supplementary information, Figure S1D and S1E).

\section{p53 is upregulated and specifically accumulated in the nucleolus in the def ${ }^{\text {hit29 }}$ mutant}

We demonstrated previously that the transcriptional expression of $\triangle 113 p 53$ is totally p53 dependent and that the transcript level of $\triangle 113 p 53$ was greatly elevated in the $d e f^{h i 429}$ mutant $[13,17]$. Interestingly, the transcript level of p53 was not obviously affected in the def ${ }^{\text {hit29 }}$ mutant, which prompted us to speculate that $\mathrm{p} 53$ protein might be stabilized or become more active in the mutant to activate the expression of $\triangle 113 p 53$. Western blot using a monoclonal antibody that detects both p53 and $\Delta 113 p 53$ of zebrafish showed that both proteins were upregulated in the $d e f^{\text {hit29 }}$ mutant at $5 \mathrm{dpf}$ (Figure 1A). The def-specific morpholino (def-MO) could knock down Def protein expression in zebrafish by targeting the def gene at its splicing junction of exon 2 and intron 2 [13]. Western blot showed that $\mathrm{p} 53$ and $\Delta 113 \mathrm{p} 53$ were upregulated in the def-MO morphants at $3 \mathrm{dpf}$ (Supplementary information, Figure S1F and S1G). Immunostaining using a polyclonal antibody that recognizes both p53 and $\Delta 113$ p53 of zebrafish revealed a clear and strong positive signal colocalized with Fib-labeled nucleoli in the cells of the intestinal epithelium of $4 \mathrm{dpf} d e f^{h i 429}$ mutants but not in those of wild-type zebrafish (Figure 1B; Supplementary information, Figure S2A and S2B). Knockdown of $\Delta 113 \mathrm{p} 53$ by its specific morpholino $\Delta 113 p 53-\mathrm{MO}$ [17] had no obvious effect, while knockdown of p53 by its specific morpholino, $p 53-\mathrm{MO}^{\mathrm{ATG}}$, almost abolished the nucleolar staining pattern of p53 in def ${ }^{h i 429}$ mutants (Figure 1B). Therefore, the loss of function of def upregulated p53 protein expression, and p53 protein accumulated in the nucleoli in the def $f^{\text {hit29 }}$ mutant cells. As $\Delta 113$ p53 can form a complex with p53 [17], we speculated that the upregulated $\Delta 113 \mathrm{p} 53$ protein probably accumulates, together with p53, in the nucleoli of the mutant cells, although further concrete evidence is needed to prove this hypothesis.

\section{Def selectively triggers the degradation of p53 and} $\triangle 113 p 53$ protein

The above results suggested that Def regulates the stability of $\mathrm{p} 53$. Indeed, we found that co-injection of $M y c-d e f$ but not of Myc-def ${ }^{\text {ttop }}$ (a def mutant that harbors a premature stop codon at codon 55 created by site-directed mutagenesis) [13] mRNA drastically reduced the level of p53 protein (Figure 1C, protein panels) but not that of p53 mRNA (Figure 1C, RNA panels) at $6 \mathrm{~h}$ postinjection (hpi). In fact, Def reduced the level of p53 as early as 1 hpi (Supplementary information, Figure S2C). To our surprise, overexpression of Def also reduced the level of HA- $\Delta 113 \mathrm{p} 53$ protein (Figure 1D, protein panels) but not that of mRNA (Figure 1D, RNA panels) at 6 hpi. To determine whether Def reduced the level of p53 selectively, we replaced $p 53$ mRNA with egfp (enhanced green fluorescent protein) or $\mathrm{rcll}$ (encoding a nucleolar protein) mRNA and found that $M y c-d e f$ did not affect the protein levels of EGFP (Figure 1E) or Rcl1 [24] (Supplementary information, Figure S2D). Interestingly, Def failed to reduce the protein levels of two p53 mutants, R143H and R250W (Figure 3D). As R250W and R143H differ from $\mathrm{p} 53$ only by an $\mathrm{A}^{748}$ to $\mathrm{T}^{748}$ single nucleotide change and by an $\mathrm{R}^{143}$ codon AGA to $\mathrm{H}^{143}$ codon CAC change in the $\mathrm{p} 53$ coding region, respectively, which are relatively distant from the translation start codon ATG, we therefore concluded that Def overexpression selec- 
A

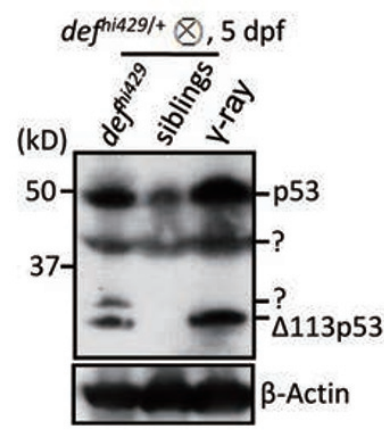

B

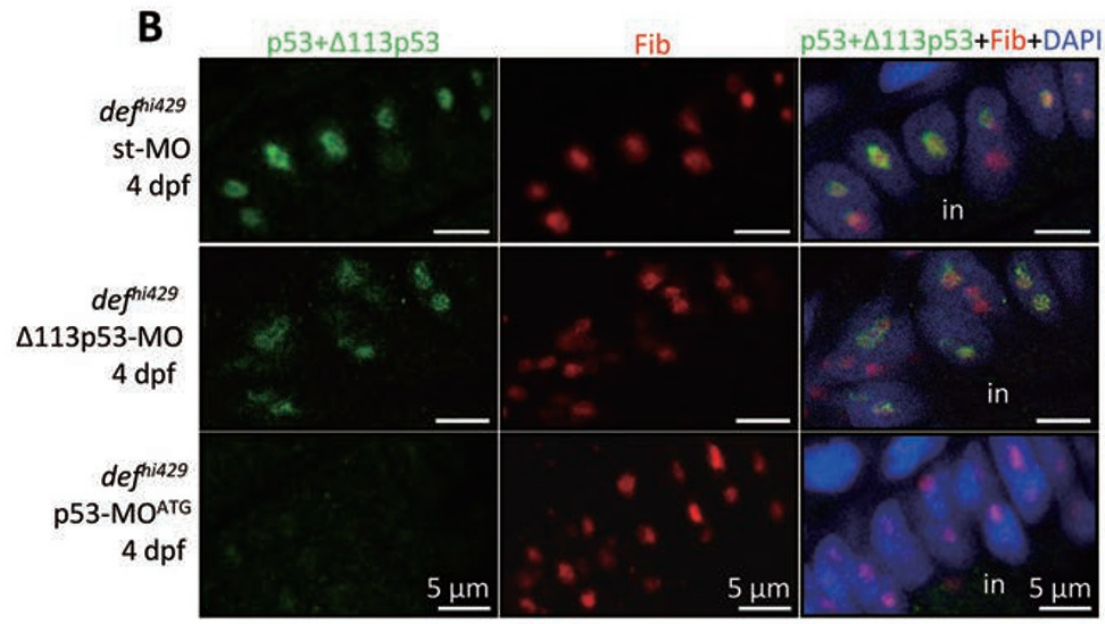




Figure 1 Def selectively induced the degradation of p53 and $\Delta 113 p 53$ proteins. (A) Western blot of p53 and $\Delta 113 p 53$ using the A7-C10 monoclonal antibody to detect both proteins in def ${ }^{\text {hi429 }}$ homozygotes and non-homozygous siblings at 5 dpf and in $\gamma$-ray-treated wild-type embryos. ?, uncharacterized p53 isoforms; $\beta$-actin, loading control. (B) Coimmunostaining of Fib and p53/ $\Delta 113 p 53$ in a def ${ }^{\text {hi429 }}$ mutant embryo injected with st-MO (upper panel), $\Delta 113 p 53-\mathrm{MO}$ (middle panel) or $p 53-\mathrm{MO}^{\mathrm{ATG}}$ (bottom panel) morpholinos at $4 \mathrm{dpf}$. Nuclei were stained with DAPI. st-MO: standard control morpholino. in: intestine. (C) Western blot (top three panels) of p53 protein and northern blot (bottom two panels) of p53 mRNA in embryos injected with different mRNA mixes at 6 hpi as shown. 28S rRNA: RNA loading control. GAPDH, protein loading control. (D) Same as in (C), but analysis of $\Delta 113 p 53$. (E) Same as in (C), but analysis of EGFP. (F) tp53 ${ }^{M 214 K}$ mutant embryos were injected with different mRNA mixes or phenol red dye. The survival rate of embryos in each treatment group at 12 hpi was analyzed. The values plotted represent mean \pm SEM (three repeats of $n=100-200$ embryos each), with test $P$-values indicated. (G) Analysis of apoptosis in embryos described in (F) at 10 hpi. Embryos were categorized based on their number of apoptotic cells. Category $1,<50$ apoptotic cells per embryo; category 2, between 50-300 apoptotic cells per embryo; category 3 , > than 300 apoptotic cells per embryo. Graphics shows the number of embryos in each category in each case. (H) qPCR analysis of p53 target genes in embryos described in (F). The qPCR values were normalized against elf1a and expressed as fold change in expression. The values plotted represent mean \pm SEM. The $P$-value was obtained by performing the two-tailed unpaired $t$ test. ${ }^{* *} P<0.001 ;{ }^{* *} P<0.01$. 
tively promotes the degradation of $\mathrm{p} 53$ and $\Delta 113 \mathrm{p} 53$ protein without affecting their mRNA stability or protein translation in zebrafish.

Eight def deletion constructs (Supplementary information, Figure S3A) were generated and each in vitro synthesized mRNA was co-injected with HA-p53 mRNA. The level of HA-p53 was then analyzed at 6 hpi. Deletion of the first 189 (construct D1) or 377 (construct D2) amino acids (AAs) from the N-terminus, which contains the NoLS, almost totally abolished the ability of Def to promote HA-p53 degradation. In contrast, deletion of the last 376 AAs (constructs D3,D4 and D5) from the C-terminus did not affect Def-mediated p53 degradation (Supplementary information, Figure S3B). Surprisingly, removal of the last 64 AAs (construct D6) inactivated the Def protein, whereas deletion of AAs 566-627 (construct D7) caused a moderate loss of Def activity. We also deleted the internal region between AAs 628 and 689 (construct $D 8$ ) and found that the resultant protein was more effective in promoting HA-p53 degradation than the full-length Def (Supplementary information, Figure S3C). Thus, we concluded that the NoLS is essential for the activity of Def on p53. Meanwhile, we identified two promoting regions (AAs 1-189 and 566-627) and one repressing region (AAs 628-689) in the Def protein function, suggesting a highly regulated and complex mechanism of action in Def-mediated p53 degradation.

\section{Def protects the survival of embryos by antagonizing $p 53$ apoptotic activity}

The zebrafish mutant $t p 53^{\mathrm{M} 214 \mathrm{~K}}$ carries a $\mathrm{M}^{214}$ to $\mathrm{K}^{214}$ substitution in the p53 DNA-binding domain that creates a loss-of-function allele of $p 53$ [25]. Injection of the normal $p 53$ mRNA alone caused high embryo mortality $(\sim 70 \%)$ at 12 hpi in the $t p 53^{\mathrm{M} 214 \mathrm{~K}}$ mutant background. Co-injection of def and $p 53$ mRNAs greatly reduced the embryo mortality seen following injection of $p 53$ mRNA alone (Figure 1F; Supplementary information, Figure S2E and Table S1). The terminal deoxynucleotidyl transferase dUTP nick end labeling (TUNEL) assay showed that injection of the $p 53 \mathrm{mRNA}$ alone caused massive cell apoptosis, and that injection of def but not of def top mRNA strongly inhibited p53-induced apoptosis in the co-injected embryos at 10 hpi (Figures $1 \mathrm{G}$; Supplementary information, Figure S2F and Table S2). Concomitantly, compared with their expression in embryos injected with the $p 53 \mathrm{mRNA}$ alone, the expression of all p53 target genes, including $\triangle 113 p 53, m d m 2, p 21$ and bax, was downregulated by the injection of def mRNA in the co-injected embryos at 6 hpi (Figure $1 \mathrm{H}$ and Supplementary information, Table S3).
Def-mediated degradation of p53 and $4113 p 53$ is independent of $M d m 2$

$\Delta 113$ p53 lacks the motif essential for Mdm2 binding [26]. Mdm2 binds to the N-terminus of p53 and targets the C-terminus of $\mathrm{p} 53$ for ubiquitination, a prerequisite for $\mathrm{p} 53$ degradation through the $26 \mathrm{~S}$ proteasome [2]. We therefore predicted that $\Delta 113 \mathrm{p} 53$ would be resistant to Mdm2-mediated protein degradation. We co-injected $M y c-d e f$ or $M y c-m d m 2$ mRNA with HA-4113p53 or $H A-p 53$ P5 mRNA (similarly to $\Delta 113 \mathrm{p} 53$, P5 lacks the Mdm2-binding motif) and found that Def but not Mdm2 promoted the degradation of both $\Delta 113 \mathrm{p} 53$ and p53_P5 at 6 hpi (Figure 2A). In addition, knockdown of Mdm2 by an $m d m 2$-specific morpholino ( $m d m 2-\mathrm{MO})$ did not prevent the degradation of $\mathrm{p} 53$ by Def in the injected embryos, and vice versa (Supplementary information, Figure S4). Mdm2 is the key E3 ligase that mediates the degradation of $\mathrm{p} 53$ through the $26 \mathrm{~S}$ proteasome pathway, and its depletion causes embryo lethality in zebrafish [27]. We used $m d m 2-\mathrm{MO}$ to knock down $\mathrm{Mdm} 2$ protein expression and found that the level of endogenous p53 protein was greatly elevated in the $m d m 2-\mathrm{MO}$ morphants (Figure 2B). We co-injected def mRNA and $m d m 2-\mathrm{MO}$ and found that Def reduced the level of p53 protein in the $m d m 2-\mathrm{MO}$ morphants at both 10 and 24 hpi (Figure $2 \mathrm{~B})$, concomitant with a reduction in their apoptosis at 24 hpi (Figure 2C). These results demonstrated that Def promotes the degradation of both p53 and $\Delta 113 \mathrm{p} 53$ in an Mdm2-independent manner.

\section{Def targets the p53 DNA-binding domain for degrada-} tion

Six $p 53$ deletion constructs $(P 1-P 6)$ (Figure $3 \mathrm{~A})$ were generated for the identification of the Def-targeting domain(s). Def only promoted the degradation of those deletion products that contained the p53 DNA-binding domain $(P 2, P 4$ and $P 5)$ at 6 hpi (Figure 3B). Noteworthy, Mdm2 triggered the degradation of the $P 6$ product only, but had no or only a weak effect on the products that were truncated from the $\mathrm{N}-(P 2, P 3$, and $P 5)$ or $\mathrm{C}$ terminus ( $P 2$ and $P 4)$ of $\mathrm{p} 53$ (Figure $3 \mathrm{C}$ ). This is consistent with earlier studies that showed that both the Nterminal Mdm2-binding domain and the C-terminal ubiquitin target sites of p53 are requisite for the Mdm2driven degradation of p53 [26].

Three zebrafish p53 mutants were created, each with a point mutation that alters a crucial functional residue, including R143H, R250W and R313C that are counterparts of human hu-p53-R175H, -R282W and -R337C, respectively. hu-p53-R175H and $-\mathrm{R} 282 \mathrm{~W}$ are known to lose DNA-binding capacity [28] whereas hu-p53-R337C displayed the highest DNA-binding activity [29]. We 

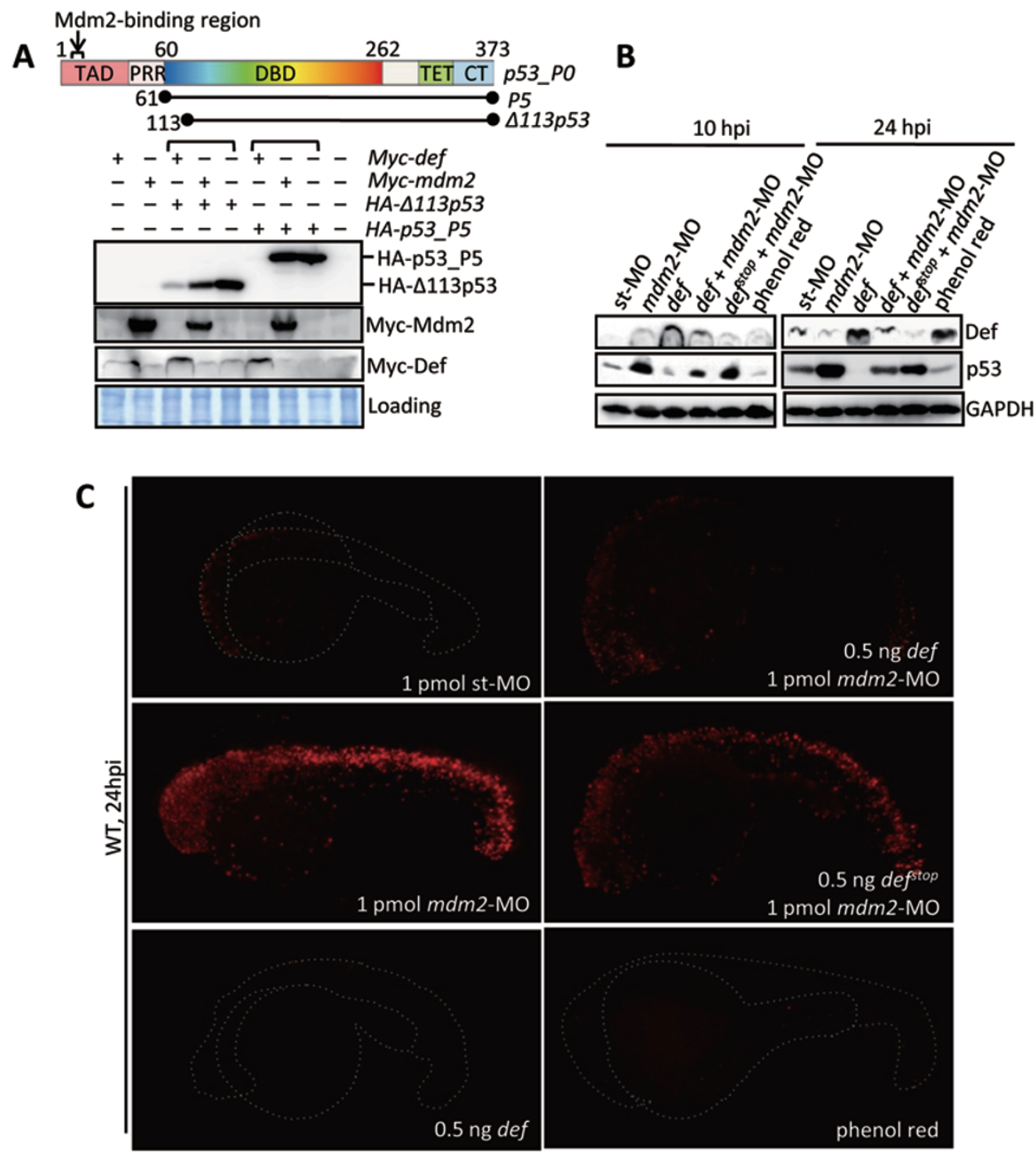

Figure 2 Def-mediated p53 degradation was independent of Mdm2. (A) Diagram on the top shows the Mdm2-binding domain and other key functional domains of the p53 protein, and shows the truncated sites for $\triangle 113 p 53$ and p53-P5. TAD, transactivation domain; PRR, proline-rich region; DBD, DNA-binding domain; TET, tetramerization domain; CT, C-terminus regulation domain. Numerical numbers denote the amino acid position from Met. Western blot analysis of p53, Def and Mdm2 examined the effects of Def and Mdm2 on the stability of $\Delta 113$ p53 and p53-P5 proteins at 6 hpi. Commassie Blue staining, protein loading control. (B, C) Western blot of p53 and Def for analyzing the effect of Def on endogenous p53 induced by $\mathrm{mdm2-MO}$ at 10 hpi and $24 \mathrm{hpi}$, respectively (B). TUNEL assay for analysis of apoptosis in embryos injected with $m d m 2-\mathrm{MO}$ alone or $m d m 2-$ $\mathrm{MO}+$ def mRNA mix at $24 \mathrm{hpi}(\mathbf{C})$. st-MO morpholino, def ${ }^{\text {stop }}$ mRNA and phenol red dye injections were used as the controls.

found that although Mdm2 triggered the degradation of all these three p53 mutant proteins, Def was unable to promote the degradation of zebrafish zf-p53-R143H and -R250W but promoted zf-p53-R313C degradation at 6 hpi (Figure 3D). Therefore, Def-induced p53 degradation is dependent on the molecular status of the p53 protein that requires a wild-type DNA-binding domain.
Def-mediated p53 degradation is independent of the ubiquitination pathway

In addition to Mdm2, many other E3 ligases have been found to trigger the degradation of $\mathrm{p} 53$ through the ubiquitination pathway [2]. We injected one-cell stage zebrafish embryos with HA-ubiquitin mRNA and found that this promoted degradation of p53 in a dosage-dependent manner at 6 hpi (Figure 3E). Multiple lysine residues 
A

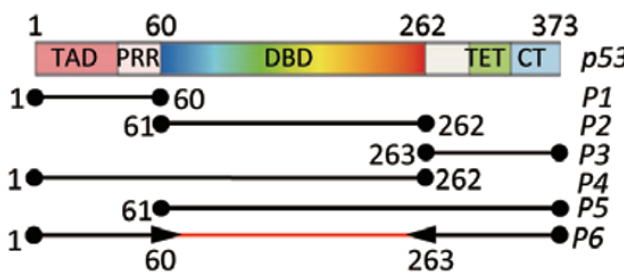

B

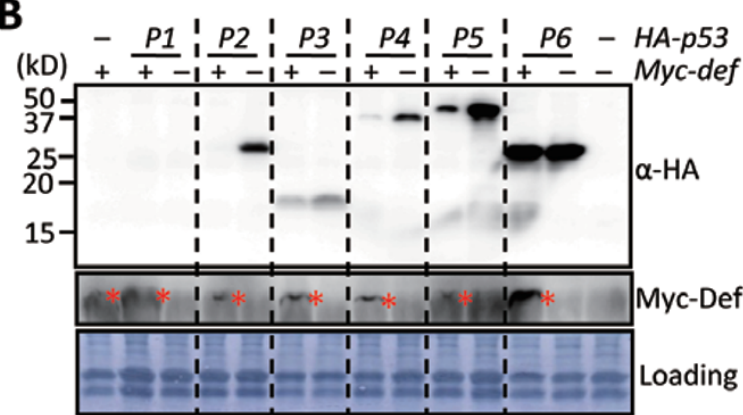

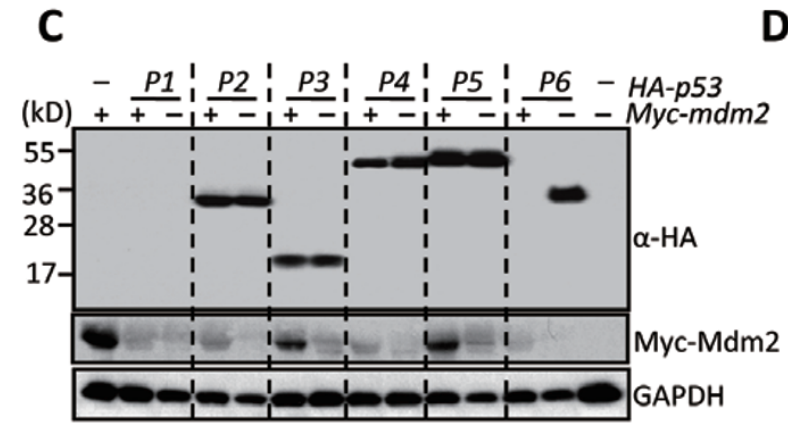

C
F -++++ p53-9KR

-++++ Myc-mdm2

- - 100200400 HA-ub (pg)

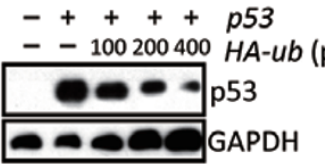

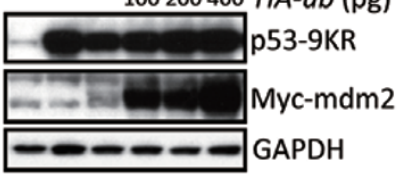

Figure 3 Def-mediated p53 degradation is independent of the ubiquitination pathway. (A) Diagram shows the structure of p53 protein (refer to Figure 2A) and six p53 deletion mutant constructs. (B) Western blot of p53, six p53 deletion mutant products and Def for analyzing the effect of Def on their stability in the co-injected embryos at 6 hpi. (C) Same as in (B) but analyzing the effect of Mdm2. (D) Western blot of p53, Def and Mdm2 for comparing the effect of Def and Mdm2 on the stability of p53 mutant proteins R143H, R250W and R313C in the injected embryos at 6 hpi. (E) Western blot of p53 for comparing the effect of different dosages of ubiquitin on the stability of p53 in the injected embryos at 6 hpi. (F) Western blot of p53 and Mdm2 for analyzing the effect of Mdm2 plus ubiquitin on the stability of p53 mutant protein p53-9KR in the injected embryos at 6 hpi. (G) Same as in (F) but analyzing the effect of Def.

in the p53 protein are known to serve as the sites for ubiquitination [26]. We selected nine lysine $(\mathrm{K})$ residues $\left(\mathrm{K}^{292}, \mathrm{~K}^{293}, \mathrm{~K}^{295}, \mathrm{~K}^{345}, \mathrm{~K}^{347}, \mathrm{~K}^{348}, \mathrm{~K}^{360}, \mathrm{~K}^{361}\right.$, and $\left.\mathrm{K}^{365}\right)$ and mutated them to arginine $(\mathrm{R})$ (construct $z f-p 53-9 K R$ ). We co-injected $z f-p 53-9 K R$ with $M y c-m d m 2$ or def mRNA together with HA-ubiquitin mRNA. The results showed that zf-p53-9KR was totally stable after the co-injection of Mdm2 with $400 \mathrm{pg}$ HA-ubiquitin mRNA (Figure 3F) but was degraded by Def with or without HA-ubiquitin at 6 hpi (Figure 3G). Thus, Def-mediated p53 degradation occurs via an ubiquitination-independent pathway.
Two naturally occurring single-nucleotide polymorphisms (SNPs) in hu-def alter hu-Def activity on p53

Before studying hu-Def, we sequenced $h u$-Def cDNA cloned from different human cell lines including MCF7, HCT116, U2OS, HepG2, SiHa, Ovcar-8/TR, PC-3 and Saos-2 and searched the dbSNP database (http://www. ncbi.nlm.nih.gov/snp/). We identified two SNPs (G/C at position $\mathrm{ORF}^{199}$, converting $\mathrm{E}^{67}$ to $\mathrm{Q}^{67}$, and $\mathrm{A} / \mathrm{G}$ at position $\mathrm{ORF}^{332}$, converting $\mathrm{D}^{111}$ to $\mathrm{G}^{111}$ ) in its coding region (Figure 4A). We found that overexpression of the products encoded by all four combinations of these two SNPs in MCF7 cells downregulated the levels of hu-p53 (Figure $4 \mathrm{~B}$ ) and $\Delta 133 \mathrm{p} 53$ (Figure $4 \mathrm{C}$ ) at $24 \mathrm{~h}$ post-transfection 
A

\begin{tabular}{|l|l|l|l|l|l|l|l|l|l|l|}
\hline Cell line & MCF-7-1 & MCF-7-2 & MCF-7-3 & HCT-116 & U2OS & HepG2 & SiHa & OVCAR-8/TR & PC-3 & Saos-2 \\
\hline hu-def ORF199 & C/G & G/G & C/G & C/G & C/G & C/G & C/G & G/G & G/G & C/G \\
\hline hu-def ORF332 & G/G & A/A & G/G & G/G & G/G & G/G & G/G & G/G & G/A & G/G \\
\hline
\end{tabular}

B

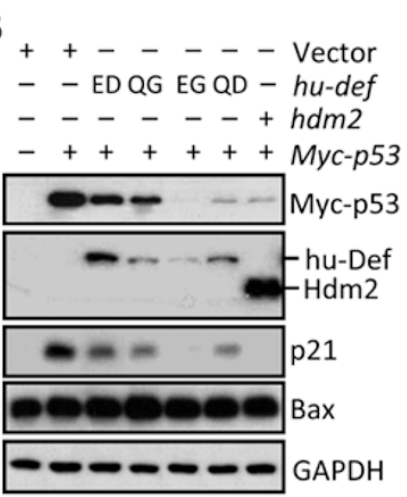

C

++-- - Vector

- EDQG EG QD hu-def

-+++++ Myc- $-133 p 53$

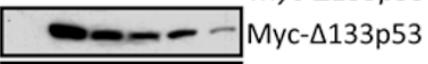

$\longrightarrow$ hu-Def

GAPDH

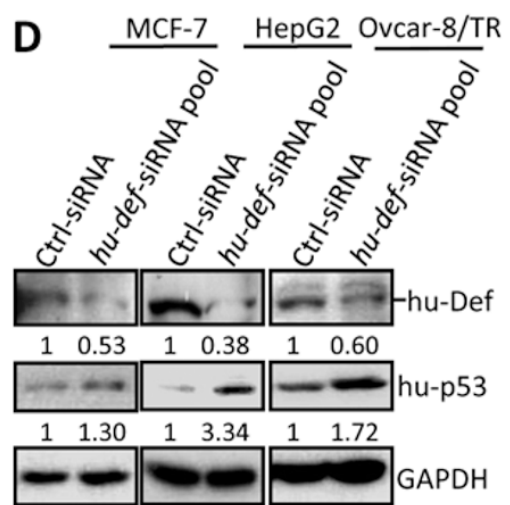

$E$

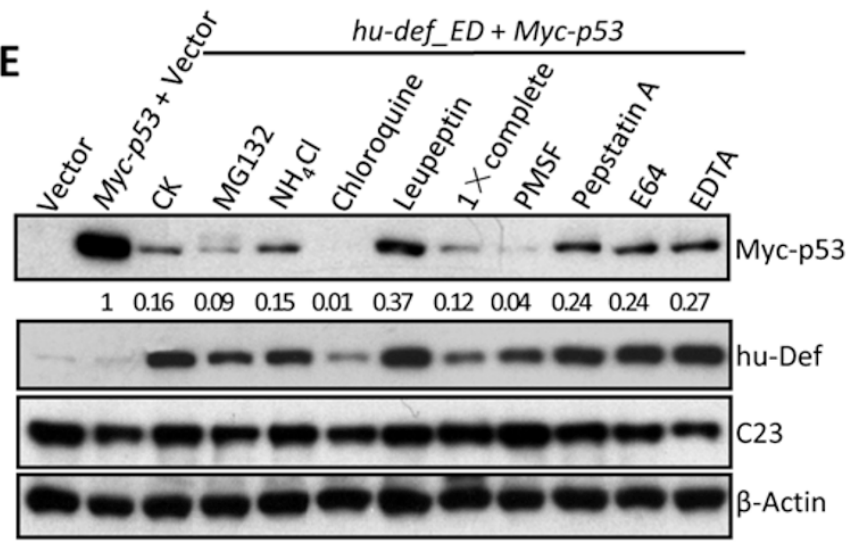

$\mathbf{F}$

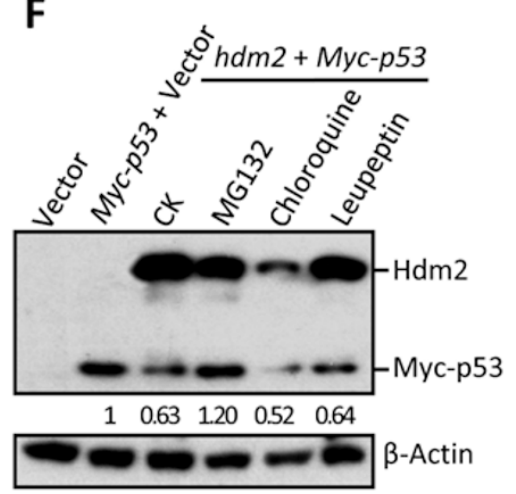

Figure 4 Def-mediated p53 degradation was inhibited by the cysteine protease inhibitor. (A) Sequencing genomic DNA from different human cells identified two SNPs (G/C at codon position ORF ${ }^{199}, \mathrm{~A} / \mathrm{G}$ at codon position ORF ${ }^{332}$ ) in the hu-Def coding region. (B) Western blot of p53, Def, Hdm2, p21 and Bax for comparing the effect of hu-Def encoded by four combinations of $(\mathrm{G} / \mathrm{C})^{199}$ and $(\mathrm{A} / \mathrm{G})^{332}$ SNPs on the stability of p53 in MCF-7 cells co-transfected with MYC-tagged p53 plasmid, and the expression of p53 target genes p21 and Bax at $24 \mathrm{hpt}$ (C) Same as in (B) but analysis of $\Delta 133 p 53$ in co-transfected cells. (D) Western blot of p53 and Def in MCF-7, HepG2 or Ovcar-8/TR cells treated with def specific siRNA pool (hu-def-siRNA pool) at $24 \mathrm{hpt}$. (E) Western blot of p53 and Def for comparing the effect of various inhibitors on hu-Def-mediated p53 degradation. (F) Same as in (E) but analysis of the effect of selected inhibitors on Hdm2-mediated p53 degradation. In (D-F), fold changes in expression for p53 or hu-Def against their respective controls (set as 1) are shown under their corresponding panels. GAPDH or $\beta$-actin was used as the normalization control.

(hpt). Interestingly, the products of the combination of $\mathrm{G}^{199} \mathrm{G}^{332}$ (hu-Def_EG) and $\mathrm{C}^{199} \mathrm{~A}^{332}$ (hu-Def_QD) exhibited much higher activity than those of $\mathrm{G}^{199} \mathrm{~A}^{332}$ (hu-Def ED) and $\mathrm{C}^{199} \mathrm{G}^{332}$ (hu-Def_QG) in the degradation of $\mathrm{p} 5 \overline{3}$ (Figure 4B) and $\Delta 133 \mathrm{p} 53$ (Figure $4 \mathrm{C}$ ). Examination of the p53 target genes showed that p21 but not Bax was downregulated in the $h u$-def and p53 co-transfected cells (Figure 4B). In contrast, we found that the knockdown of hu-Def by its specific small interfering RNA (siRNA) upregulated the level of endogenous p53 protein in MCF-7, HepG2 and Ovcar-8/TR cells (both MCF-7 and HepG2 cells carry wild-type p53 whereas Ovcar-8/TR has a mutated p53 with an in-frame deletion of 126-132 AAs) at $24 \mathrm{hpt}$ (Figure 4D).

Def-mediated p53 degradation is dependent on Calpain 3

We then tested the effects of various protein degradation inhibitors on Def-mediated p53 degradation. Only 

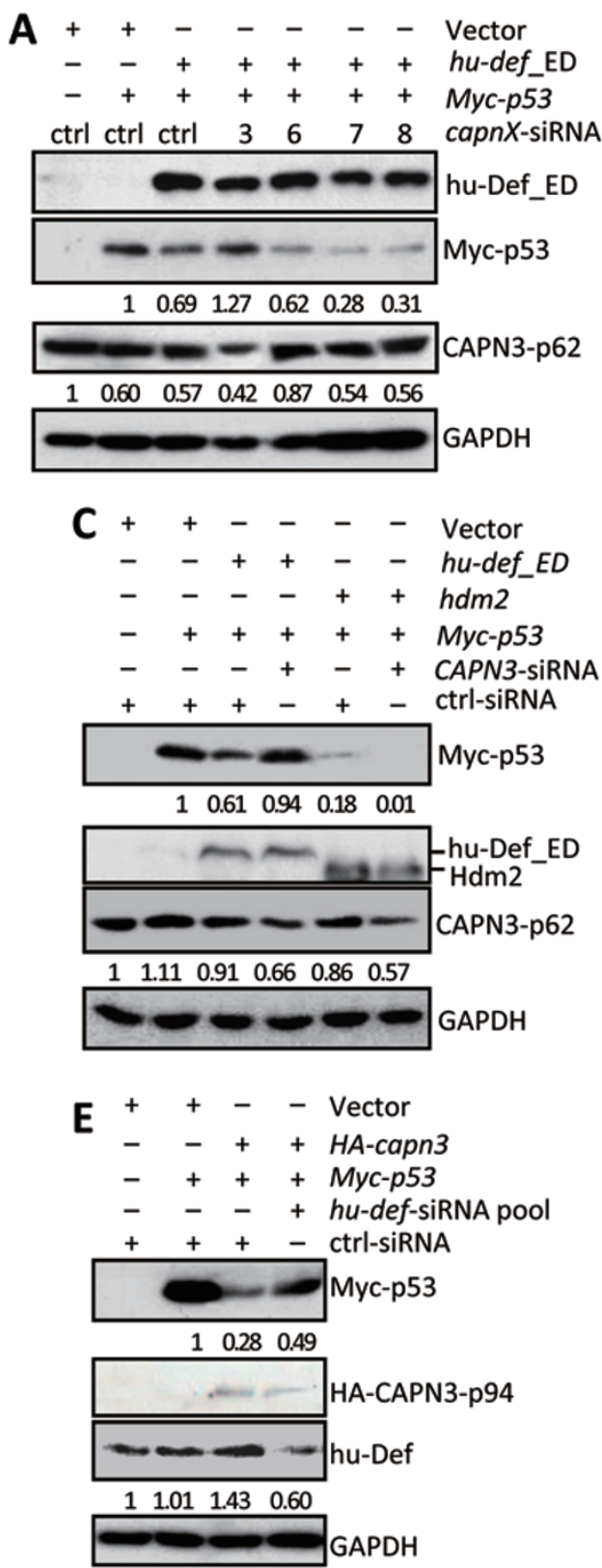

B
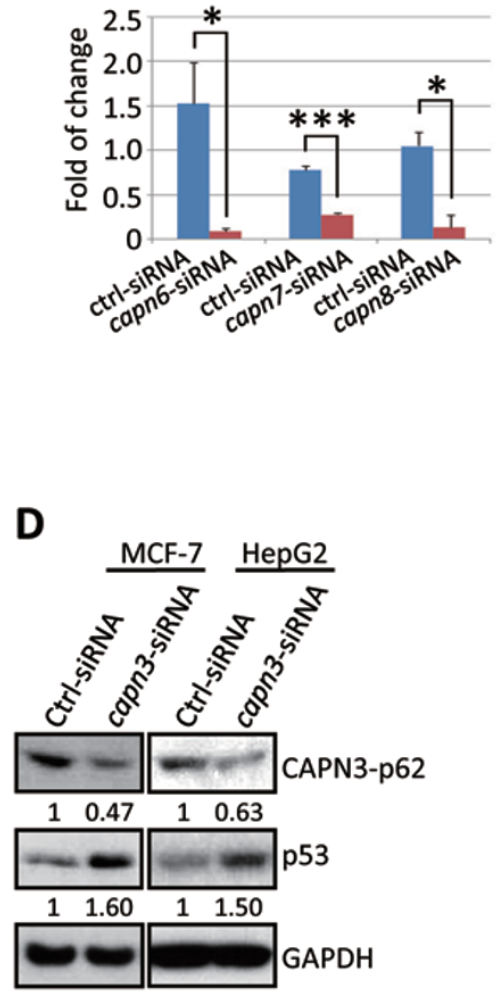

Figure 5 Def-mediated p53 degradation depends on cysteine protease, CAPN3. (A) Western blot of p53 and Def for comparing the effect of knockdown of CAPN3, $-6,-7$ and -8 by their specific siRNAs on hu-Def-mediated p53 degradation. (B) qPCR showing the efficiency of knockdown of capn6, capn7 and capn8 by their respective siRNAs. The relative expression level of the genes was shown in fold change as normalized against human elf1a. (C) Western blot of p53, Def, Hdm2 and CAPN3 in MCF-7 cells treated with CAPN3-siRNA. (D) Western blot of p53 and CAPN3 in CAPN3-siRNA treated MCF-7 and HepG2 cells at $24 \mathrm{hpt}$. (E) Western blot of p53, CAPN3 and Def in cells transfected with CAPN3, p53 overexpression plasmids and hu-Def siRNA. (F) Co-IP analysis of interaction between hu-Def and CAPN3 in H1299 cells. Cells were transfected with plasmids as indicated. In (A, and $\mathbf{C}-\mathbf{E})$, fold changes in expression for p53, hu-Def or CAPN3 against their respective controls (set as 1) are shown under their corresponding panels. GAPDH was used as the normalization control.

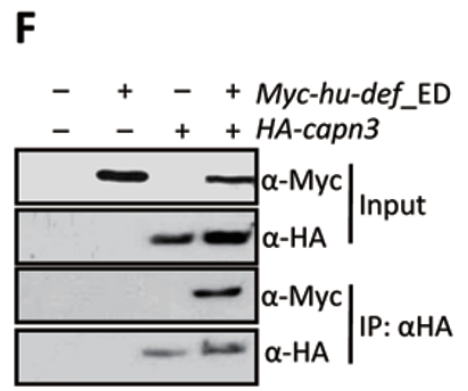

cysteine protease inhibitors (leupeptin, pepstatin A and E64) and EDTA, but not inhibitors of the 26S proteasome (MG132), lysosomes $\left(\mathrm{NH}_{4} \mathrm{Cl}\right.$ and chloroquine) or autophagy (3-methyladenine, data not shown), inhibited Def-mediated p53 degradation (Figure 4E). In contrast, although MG132 showed a strong inhibitory effect, leupeptin showed only a negligible effect on Hdm2-mediated p53 degradation (Figure 4F). This result also ruled out the possibility of the involvement of free $20 \mathrm{~S}$ proteasome in Def-mediated degradation of $\mathrm{p} 53$ because MG132 was also an effective inhibitor of this process. Interestingly, hu-p53-R175H, which is poorly sensitive to Calpain (a type of cysteine proteases) [4], was more resistant to Def-mediated degradation (Supplementary information, Figure S5).

The above results suggested that Def and Calpain might act together to mediate p53 degradation. Humans have 15 known calpain (capn) genes [30]. We designed specific siRNAs against these 15 human capn genes (Supplementary information, Table S4) and used them to knockdown the expression of their corresponding CAPNs. We then tested the effect of hu-Def on p53 in cells treated with each siRNA. We found that only the knockdown of CAPN3 expression, but not 
that of CAPN6, -7 or -8 (Figure 5A and 5B) or others (Supplementary information, Figure S6A), blocked Defmediated p53 degradation. In contrast, the knockdown of CAPN3 expression did not block Hdm2-mediated p53 degradation (Figure 5C). We then determined the effect of the knockdown of CAPN3 on p53 and found that it significantly increased the level of endogenous p53 protein at 24 hpt in MCF7 and HepG2 cells (Figure 5D). Therefore, Def-mediated p53 degradation was CAPN3 dependent. More importantly, because CAPN3 is the only CAPNs known to date to be localized in the nucleolus [31], the above results strongly suggested that the Def- and CAPN3-mediated p53 degradation pathway operates in the nucleolus.

\section{hu-Def and CAPN3 form a complex}

As shown above, CAPN3 knockdown blocked hu-Defmediated p53 degradation (Figure 5A-5C). To determine whether hu-Def knockdown could block CAPN3-mediated p53 degradation, MCF7 cells were cotransfected with
A

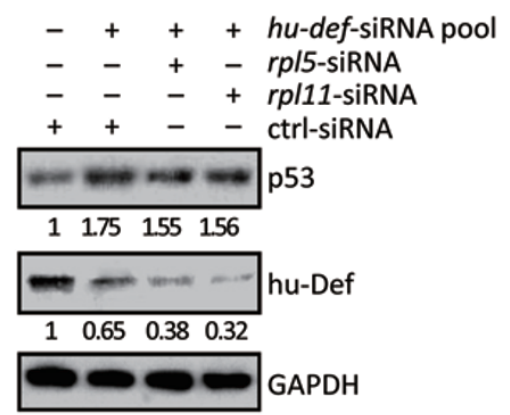

B

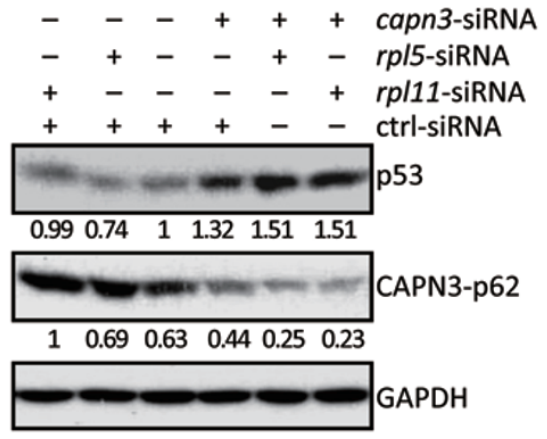

C

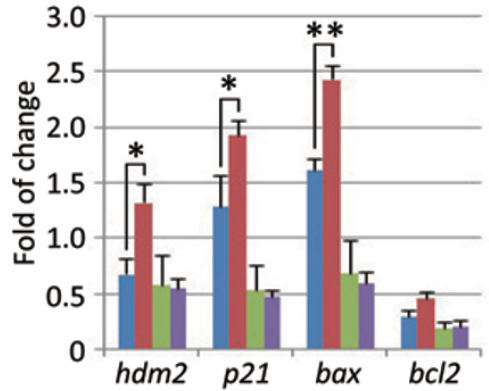

= HepG2|ctrl-siRNA

- HepG2 |capn3-siRNA

= $\mathrm{H} 1299 \mid$ ctrl-siRNA

- H1299|capn3-siRNA

D


Figure 6 CAPN3 knockdown activated p53 response. (A, B) Western blot analysis of the effect of RPL5 or RPL11 knockdown on the stabilized p53 induced by Def (A) or CAPN3 (B) knockdown. Fold changes in expression for $p 53$, huDef or CAPN3 against their respective controls (set as 1) are shown under their corresponding panels. GAPDH was used as the normalization control. (C) qPCR analysis of expression of p53 response genes in HepG2 or H1299 cells treated with control siRNA (ctrl-siRNA) or capn3siRNA. The relative expression level of the genes is shown in fold change as normalized against human elf1a. (D) FACS analysis of the ratio of cells at G2/ $\mathrm{M}, \mathrm{S}$ and $\mathrm{G} 1$ phases (histogram on the left) after cells were treated with ctrl-siRNA (middle graph) or capn3-siRNA (graph on the right). (E) Analysis of apoptotic cells (histogram on the left) after Annexin $V$ staining on cells treated with ctrl-siRNA (middle graph) or capn3-siRNA (graph on the right). 
CAPN3 overexpression plasmid and hu-Def siRNA, and p53 protein was analyzed. Western blot results showed that hu-Def knockdown clearly partially blocked the effect of CAPN3 on p53 (Figure 5E). Apparently, Def and CAPN3 are mutually dependent for the maximization of their effect on p53. We next overexpressed Myc-tagged hu-Def and HA-tagged CAPN3 together in H1299 (p53null) and MCF7 (p53 wild type) cells and performed coimmuoprecipitation (Co-IP) using the anti-HA antibody. Western blot of Co-IP products using the anti-Myc antibody showed that hu-Def was successfully coprecipitated with CAPN3 (Figure 5F; Supplementary information, Figure S6B). This result demonstrated that Def and CAPN3 can form a complex even in the absence of $\mathrm{p} 53$.

The Def-CAPN3 pathway is independent of the RPL5/ RPL11 pathway

Previous studies have shown that the ribosomal proteins, RPL5 and RPL11, stabilize and activate p53 by sequestrating MDM2 to the nucleolus under conditions of nucleolar stress $[10,11]$. Knockdown of RPL5 or RPL11 would leave MDM2 free in the nucleoplasm, which would trigger the degradation of p53 even under nucleolar stress [12]. Our extensive data shown above demonstrated that Def-mediated p53 degradation is independent of the Mdm2 pathway. To further verify this conclusion, we tested the effect of the combined knockdown of Def and RPL5, Def and RPL11, CAPN3 and RPL5, and CAPN3 and RPL11 by their respective siRNAs. Clearly, the elevated levels of p53 induced by Def or CAPN3 knockdown were not affected by the concomitant knockdown of either RPL5 or RPL11 (Figure 6A and 6B).

Knockdown of CAPN3 arrests cells at the G1 phase and promotes cell apoptosis

We then performed qPCR to examine the expression of p53 reponse genes, including $h d m 2, p 21$, bax and bcl2 in HepG2 (p53 wild type) and H1299 cells after treatment with CAPN3 siRNA at $24 \mathrm{~h}$. We found that $h d m 2$ and bax were significantly upregulated in HepG2 cells but not in H1299 cells after CAPN3 knockdown (Figure $6 \mathrm{C})$. We also analyzed the ratio of cells in the G1, S and G2/M phases after CAPN3 siRNA treatment. The flow cytometry analysis (FACS) result showed that CAPN3 knockdown in HepG2 cells arrested cells at the G1 phase (an average of $74.7 \% \mathrm{G} 1$ cells in control siRNA samples versus $83.5 \%$ in CAPN3 siRNA samples) (Figure 6D). FACS analysis of apoptotic cells after Annexin V staining showed that CAPN3 siRNA-treated HepG2 cells exhibited a much higher level of apoptosis than those treated with control siRNA (Figure 6E).
Capn $3 b$ acts together with Def to mediate p53 degradation in zebrafish

A database search in the zebrafish genome assembly Zv9 identified two capn3 genes, located on chromosomes 17 (named capn $3 a$ ) and 20 (named capn $3 b$ ). Amino acid sequence alignment revealed that human CAPN3 shares $62 \%$ and $57 \%$ identity with zebrafish CAPN3a and CAPN3b, respectively (Supplementary information, Figure S7). However, only CAPN3b contains the presumed NoLS KKKxKP (Supplementary information, Figure S8A). We first examined the expression patterns of capn $3 a$ and capn $3 b$ in zebrafish embryos at 2 and 4 dpf, respectively. Whole-mount in situ hybridization (WISH) results showed that capn $3 a$ is mainly expressed in the lens at $2 \mathrm{dpf}$ as recorded in the database (database for Gene Expression, http://zfin.org), and is then enriched in the brain region between the eyes (Figure 7A). In contrast, capn $3 b$ expression is enriched in the digestive organs at both 2 and $4 \mathrm{dpf}$ and in the head region at $4 \mathrm{dpf}$ (Figure 7A), and displays a pattern resembling that observed for def [13]. To determine whether Def-mediated p53 degradation in zebrafish is also CAPN3 dependent, we co-injected def and $p 53$ mRNA with a capn $3 a-$ specific (capn3a-MO) or capn3b-specific (capn3b-MO) morpholino (Supplementary information, Figure S8B and $\mathrm{S} 8 \mathrm{C}$ ) and examined p53 protein levels in the injected embryos. The results showed that capn $3 b-\mathrm{MO}$ but not capn $3 a-\mathrm{MO}$ effectively blocked Def-mediated p53 degradation at 6 hpi (Figure 7B). Furthermore, we found that capn3b-MO but not capn $3 a-\mathrm{MO}$ also increased the level of the endogenous p53 protein as def-MO and $m d m 2-\mathrm{MO}$ at 3 dpi (Figure $7 \mathrm{C}$ ). The elevated endogenous p53 protein level could be downregulated by co-injection with Myc-capn $3 b-5 m u$ mRNA that lacks the target sequence for capn $3 b-M O$ (Figure 7D). In contrast, mutating the active site $\mathrm{Cys}^{120}$ (corresponding to $\mathrm{Cys}^{129}$ at the active site in human Capn3) [32] to a serine (Capn3b-C120S) abolished the ability of the mutant Capn 3 to downregulate the $\mathrm{p} 53$ level induced by the injection of capn $3 b$-MO (Figure 7D). This result suggested that Capn3b catalytic activity is essential for Def-mediated p53 degradation and that Capn3b itself does not simply provide a platform for $\mathrm{p} 53$ degradation by an alternative mechanism. In humans, CAPN3-C129S was found to be more stable than the wild-type CAPN3 [32]. We found that, as expected, the overexpressed zebrafish Capn3b-C120S is more stable than the wild-type Capn3b (Figure 7D).

As shown in Figure 1A, the level of p53 protein was increased in the zebrafish def ${ }^{\text {hit29 }}$ mutant. To determine whether capn $3 a$ and capn $3 b$ were expressed normally in the $d e f^{h i 429}$ mutant, we measured their expression levels in zebrafish. The qPCR results showed that capn $3 b$ but 
A

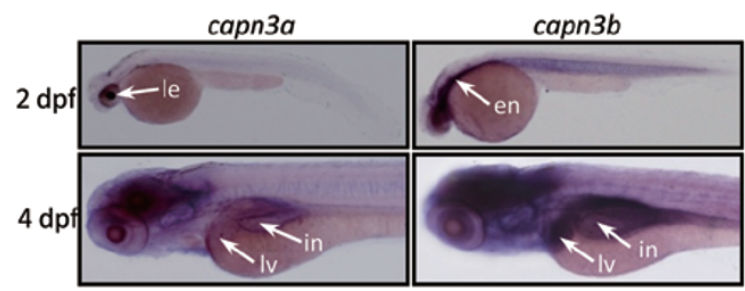

B

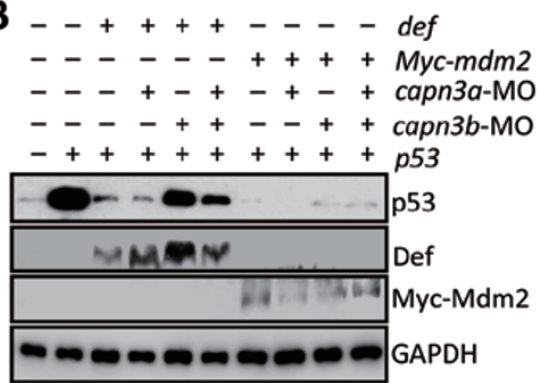

C



+ capn3b-MO

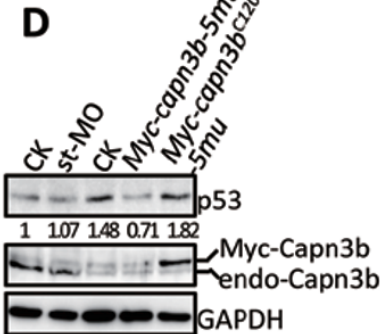



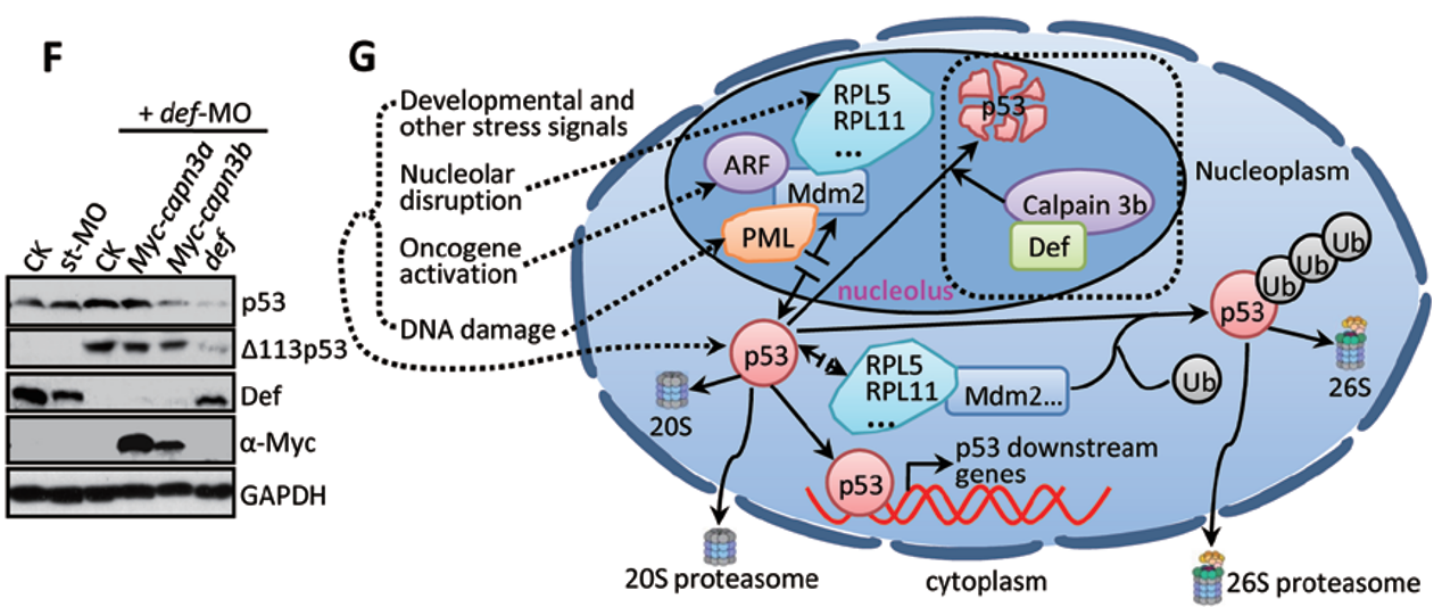

Figure 7 Def and CAPN3 duet in zebrafish. (A) WISH analysis of the expression patterns of capn3a and capn3b in embryos at 2 and $4 \mathrm{dpf}$, respectively, using capn3a or capn3b probes. en, endoderm tube; in, intestine; le, lens; Iv, liver. (B) Western blot of p53, Def and Mdm2 for comparing the effect of knockdown of zebrafish Capn3a or Capn3b with their specific morpholinos capn3a-MO or capn3b-MO on Def-mediated or Mdm2-mediated p53 degradation in the injected embryos at 6 hpi. (C) Western blot of the endogenous p53 in capn3a-MO, capn3b-MO and def-MO morphants at 3 dpi. (D) Western blot of p53 for examining the effect of capn3b mRNA lacking the capn3b-MO target sequence (Myc-capn3b-5mu) or the mutant mRNA carrying a mutation changing the codon for the active site Cys ${ }^{120}$ to $\operatorname{Ser}^{120}\left(M y c-c a p n 3 b^{C 120 S}-5 m u\right)$ on elevated endogenous p53 induced by capn3b-MO. Capn3b was detected using a polyclonal antibody against zebrafish Capn3b. Myc-Capn3b, Myctagged Capn3b; endo-Capn3b, endogenous Capn3b. (E) qPCR analysis of capn3a and capn3b transcripts in def ${ }^{\text {hi429 }}$ mutant embryos and wild-type controls (WT). The relative expression level of the genes was shown in fold change as normalized against zebrafish elf1. (F) Western blot analysis of p53, $\Delta 113 p 53$, Def and CAPN3 ( $\alpha-M y c)$ in the embryos co-injected with def-MO morphlino plus capn3a mRNA or def-MO morpholino plus capn3b mRNA at 3 dpi. Wild-type embryos (CK) and stMO-injected embryos were used as the controls. (G) Graphics summarizes the roles of the nucleolus in regulation of p53 homeostasis. In response to stress conditions such as nucleolar disruption, oncogene activation, DNA damage or developmental defect, p53 is stabilized or activated. Nucleolar factors including Arf, PML, RPL5 and RPL11 can associate with Mdm2 to prevent p53 ubiquitination and degradation through the $26 \mathrm{~S}$ proteasome in the nucleoplasm and cytoplasm. In contrast, the role of the Def-CAPN3 pathway is to prevent the accumulation of p53 in the nucleolus by triggering in situ p53 degradation.

not capn $3 a$ was expressed at a higher level in the mutant than in the wild-type control (Figure 7E), demonstrating that Capn3b-mediated p53 degradation is dependent on Def. As shown in Supplementary information, Figure 
$\mathrm{S} 1 \mathrm{~F}$, the level of $\mathrm{p} 53$ protein was elevated in the def-MO morphants. We co-injected def-MO with capn $3 a$ or cap$n 3 b$ mRNA and found that the overexpression of CAP$\mathrm{N} 3 \mathrm{~b}$ but not that of CAPN3a partially downregulated the p53 protein level (Figure 7F). It should be noted that the extent of $\mathrm{p} 53$ degradation induced by capn $3 b$ mRNA was less than that induced by $\operatorname{def}$ mRNA, demonstrating that Capn3b-mediated p53 degradation is dependent on Def, as has been observed in human cells. Therefore, the Defand Capn3b-mediated p53 degradation pathway is also functional in zebrafish.

\section{Discussion}

In this study, we demonstrated that Def, a novel nucleolar factor, can induce degradation of the p53 protein in both human and zebrafish, and that this process is independent of the proteasome pathway but is dependent on a specific nucleolus localized cysteine protease, CAPN3. Hence, we concluded that Def and CAPN3 together define a conserved nucleolar protein degradation pathway that negatively regulates the function of $\mathrm{p} 53$ by mediating its turnover (Figure 7G).

The regulation of p53 turnover is essential to maintain a low level of p53 in normal cells. Many E3 ligases have been found to mediate p53 degradation through the ubiquitination pathway [2]. The role of the nucleolus in regulating the p53 function is through the binding of Mdm2 by some nucleolar factors, such as p14ARF, RPL5 and RPL11, so that Mdm2 is physically separated from the nucleoplasmic p53. In this way, p53 degradation through the ubiquitination pathway is blocked [12, 23]. We found (1) that the upregulated p53 and possibly $\Delta 113$ p53 clearly accumulated in the nucleolus in the def $f^{\text {hi429 }}$ mutant, and (2) that RPL5 or RPL11 knockdown failed to compromise the elevated level of p53 induced by CAPN3 knockdown. These results demonstrated that the Def-CAPN3-p53 pathway differs from the p14ARF-, PML- or RPL11-mediated nucleolar pathways [8, 12, 23] (Figure 7G).

Calpains belong to the family of $\mathrm{Ca}^{2+}$-dependent cysteine proteases. Although CAPN1 and CAPN2 are considered to be ubiquitous, CAPN3 is mainly expressed in the muscle [33]. A study of a Calpain3-null knockout mouse revealed that this model exhibited a phenotype that resembles the human limb girdle muscular dystrophy type 2A (LGMD2A) due to aberrant myofibrillogenesis. CAPN3 exists in many alternatively spliced isoforms. The main isoform in muscle is p94, whereas the main isoforms in human melanoma cells are p62, hMp78 and $\mathrm{hMp} 84$ (it is proposed that $\mathrm{p} 62$ is derived from $\mathrm{hMp} 78$ and hMp84). Surprisingly, in human melanoma cells, p62 and hMp78 were located in both the cytoplasm and nucleoli [31].

It has been reported that Calpains can regulate p53 stability, however, this was attributed to cytoplasmic abundant calpains [4-7]. The nature of the nucleolar localization of CAPN3 explains why Def-mediated p53 degradation depends on the activity of CAPN3 but not that of other Calpains. Importantly, the catalytic activity of Capn $3 b$ is indispensible for p53 degradation. Furthermore, we found that Def and CAPN3 can form a complex, which is consistent with the finding that Def and CAPN3 are mutually dependent for the mediation of p53 degradation in the nucleolus. We tried different means to study the potential protein-protein interaction between Def and p53, however, we did not observe any interaction between them. It would be interesting in the future to determine whether the Def-CAPN3-p53 pathway contributes to LGMD2A in the Calpain3-null mouse model.

It appeared that Def knockdown was more effective than that of Capn3b in stabilizing p53. We speculated that an additional nucleolar factor(s) might be required for the formation of a fully functional Def-Capn3 complex. Def might serve as a scaffold protein to recruit the other factor(s). In this scenario, Def knockdown would be more effective in disrupting the complex, whereas the complex could still form after Capn3b knockdown and the residual Capn3b could still induce p53 degradation. This hypothesis might explain why p53 and $\Delta 113 \mathrm{p} 53$ are highly stabilized despite that capn 3 is highly expressed in the $d e f^{h i 429}$ mutant or overexpressed in the def-MO morphants. Certainly, the possibility of the existence of other Def effector(s) cannot be excluded. The creation of a capn $3 b$ knockout mutant by the transcription activation-like effector nuclease (TALEN) method will help to resolve this query in the future.

Previous studies showed $\Delta 113 p 53$ and $\Delta 133 p 53$ are p53 target genes and function to antagonize p53 apoptotic activity $[17,19]$. Our study also demonstrated that $\Delta 113 \mathrm{p} 53 / \Delta 133 \mathrm{p} 53$ was degraded by the Def-CAPN3 pathway when overexpressed. This is the first pathway that was found to regulate the turnover of $\Delta 113 \mathrm{p} 53 /$ $\Delta 133 \mathrm{p} 53$, although further efforts are needed to prove that this pathway also operates in vivo. The fact that Def acts on the wild-type p53, but not on point mutants p53$\mathrm{R} 143 \mathrm{H}$ and $-\mathrm{R} 250 \mathrm{~W}$, and also acts on $\Delta 113 \mathrm{p} 53 / \Delta 133 \mathrm{p} 53$ implies that $\Delta 113 \mathrm{p} 53 / \Delta 133 \mathrm{p} 53$ protein adopts much of the native structure in its truncated DNA-binding domain. Our findings might provide an explanation of why tumorigenesis occurs in certain cells/tissues even when they harbor wild-type p53. Therefore, the downregulation of hu-Def or CAPN3 in such cancer cells might be a new approach for cancer therapy. 
Clearly, the Def-Capn3-p53 pathway has an important role in protecting embryos from the $\mathrm{p} 53$-induced reduction in survival. We hypothesized that during early embryogenesis, the Def-Capn3b-p53- $\Delta 113$ p53 pathway may serve as a check point to monitor environmental changes that would allow the organism to adjust its physiological response to certain cellular stresses and thus to safeguard the proceeding of normal development. However, many questions remain unanswered. For example, how does the Def-CAPN3 pathway control the genesis of the digestive organs? Are other proteins targeted by the Def-CAPN3 pathway? Are the two SNPs in hu-def related to any disease(s)? The resolution of these questions will undoubtedly help to further our understanding of the function of the nucleolus.

\section{Materials and Methods}

\section{Zebrafish lines and maintenance}

Zebrafish were raised and maintained according to the standard procedure. The def $f^{\text {hit29 }}$ mutant line was provided by Prof Nancy Hopkins. Two pairs of primers derived from lac $Z$ and $d e f$ were used to genotype the $d e f^{h i 429}$ mutant [13]. The $p 53^{--}$mutant allele $t p 53^{M 214 K}$ line [25] was provided by Professor Thomas Look.

\section{Cell lines}

MCF-7-1, MCF-7-3, HepG2 and SiHa cells were maintained in the DMEM medium (high glucose, GIBCO), MCF-7-2 in DMEM/ F12 (1:1) medium (GIBCO), HCT-116, U2OS and Saos-2 in McCoy's 5A (modified) medium, H-1299 and OVCAR-8/TR in RPMI medium 1640 (GIBCO), and PC-3 in Ham's F12. All media were supplemented with $10 \%$ newborn calf serum (NBCS, GIBCO) with the exception of $15 \%$ NBCS for the Saos-2. Plasmids were transfected into the cell by FuGENE HD (Roche) and siRNAs with Lipofectamine 2000 (GIBCO) transfection reagent according to the manufacturer's recommendations.

\section{Phylogenetic analysis}

Human and zebrafish CAPN3 amino acid sequences were retrieved from the GenBank and Ensembl Genome Browser and aligned using the Clustal W program. The phylogenetic tree was built using the MEGA 4 program [34].

\section{Plasmid construction}

Each def and $p 53$ deletion construct was derived from $\mathrm{pCS} 2{ }^{+}$$d e f-\mathrm{D} 0$ and $\mathrm{pCS} 2^{+}-p 53-\mathrm{P} 0$, respectively. The corresponding primer pairs used to generate all relevant plasmids are listed in Supplementary information, Table S5. The method to generate the internal deletion constructs $M Y C$-def D7, MYC-def D8, def ${ }^{\text {stop }} \mathrm{pCS}^{+}$ and the internal deletion construct HA-p53_P6-pCS2 ${ }^{+}$has been described previously [17]. Zebrafish $m d m 2$ was cloned by PCR from cDNA of $1 \mathrm{dpf}$ embryos, capn $3 a$ and capn $3 b$ from cDNA of $5 \mathrm{dpf}$ embryos, using the primer pairs listed in Supplementary information, Table S5. Capn3 and hu-def_ED cDNAs were obtained from MCF7 and hu-def_QG cDNA from RKO cells, and all cDNAs were cloned into the pcDNA3. $1^{+}$vector. The $\triangle 133 p 53$ construct was subcloned from $h u-p 53$ plasmid. The $h d m 2$ and ubiquitin con- structs were provided by Dr Shuyong Lin and Dr Huizhe Huang, respectively. $z f-p 53$ 9KR, R143H, R250W, R313C, hu-def_EG, hu-def_QD, hu-p53 R175H, Myc-capn3b-5mu-pCS2 $2^{+}$and $M y c-$ capn $3 b^{C 120 S}-5 m u-p C S 2^{+}$were constructed through site-directed mutagenesis using the primer pairs listed in Supplementary information, Table S5. The 5'-untranslated regions (5' UTR) of capn $3 a$ and capn $3 b$ were cloned upstream of the egfp reporter gene to create cmv:capn3a_5'UTR-egfp and cmv:capn3b_5'UTR-egfp constructs in $\mathrm{pCS} 2^{+}$, respectively. HA or MYC tags were designed into the primers to generate the tagged constructs. For mRNA injection, mRNAs were synthesized in vitro using the mMESSAGE mMACHINE Kit (Ambion) according to the manufacturer's instructions. $1 \mathrm{nl}$ mRNA or morpholinos or plasmid containing phenol red dye was injected into zebrafish embryos at the one-cell stage.

\section{DNA, RNA and protein analysis}

The primer pair 5'-AACCTGTGGCCTAATGCCTGGGA-3' and 5'-CTGGCAGTGTTCAACCACTCTGCC-3' was used to amplify the genomic DNA fragment harboring $h u$-def from different human cells and the DNA fragment was sequenced with the primer 5'-TTAAAAGTGGGGCAGTAGCAGT-3'.

Total RNA was extracted from different samples using TRIzol reagent (Invitrogen). Probes were labeled with Digoxigenin (DIG) and northern blot hybridization was performed according to the manufacturer's instructions (Roche Diagnostics). The p53-P1 probe and egfp probe have been described previously [13]. The qPCR was performed with a CFX96 Real-Time System (Bio-Rad) using SsoFast EvaGreen Supermix (Bio-Rad). The primer pairs and detailed PCR conditions for amplifying zebrafish $\triangle 113 p 53$, $m d m 2, p 21$ and bax have been described previously [13]. The primer pairs for amplifying zebrafish capn $3 a$, capn $3 b$, def and human $h d m 2, p 21$, bax and bcl2 are listed in Supplementary information, Table S6.

The methods used for protein extraction from zebrafish embryos and from cultured cells have been described previously [17, 18]. Protein electrophoresis and western blot were performed according to the instructions provided by the manufacturers.

\section{Protease inhibitors}

Protease inhibitors MG132, chloroquine and pepstatin A were purchased from Sigma, Complete Protease Inhibitor Cocktail Tablet (EDTA-free) from Roche, and $\mathrm{NH}_{4} \mathrm{Cl}$, leupeptin, PMSF (phenylmethanesulfonyl fluoride), E64 and EDTA from Sangon (Shanghai, China).

\section{Morpholinos and siRNAs}

Morpholinos were purchased from Gene Tools (Philomath, USA). def-MO, $4113 p 53-\mathrm{MO}, p 53-\mathrm{MO}^{\mathrm{ATG}}, m d m 2-\mathrm{MO}$, and the human $\beta$-globin antisense morpholino (st-MO) were used as described previously $[13,17,27]$, capn $3 a-\mathrm{MO}$ (5'-AACCCAGACGGCGTATAGGGCATCT-3') and capn 3b-MO (5'-CTGAAAACTTCTGTTCTTCTGCCAT- $3^{\prime}$ ) were designed to target the start codon ATG of the corresponding genes. All morpholinos $(0.25 \mathrm{pmol})$ were injected into one-cell stage embryos.

Stealth C1orf107 (hu-def) siRNAs and Negative Control Duplex (control siRNA) were obtained from Invitrogen (Carlsbad, USA). The $h u$-def siRNA pool containing three duplex oligonucleotides (hu-def-siRNA-1, hu-def-siRNA-2 and $h u$-def-siRNA-3), 
CAPN1 siRNA [35], CAPN2 siRNA [36], CAPN3 siRNA, CAPN5 to CAPN16 siRNAs, RPL5 and RPL11 siRNAs [12], and the negative control duplex (control siRNA) were obtained from GenePharma (Shanghai, China). All information on the siRNA sequences is listed in Supplementary information, Table S4.

\section{Antibodies}

The zf-p53 monoclonal antibody A7-C10 and polyclonal antibody against the 113-373 aa region of p53 were raised in mouse and rabbit, respectively. Polyclonal antibody against the 34-174 aa region of hu-Def was raised in rabbit. The zf-Def rabbit polyclonal antibody has been described previously [13]. Anti-Myc mouse monoclonal (9E10) antibody was purchased from Clontech (\#631206), anti-HA mouse monoclonal (HA-7) antibody from Sigma (H3663), mouse monoclonal (38F3) antibody against human Fibrillarin (ab4566) and rabbit polyclonal antibodies against GFP (ab6556) and CAPN3 (ab38963) from Abcam. Rabbit monoclonal antibody (EPR1977Y) against GAPDH was from Epitomics (\#2251-1), and rabbit polyclonal antibody against $\beta$-actin (\#4967) was from Cell Signaling Technology. Mouse monoclonal antibodies against human p53 (DO-1, sc-126), Hdm2 (SMP14, sc-965), p21 (F-5, sc-6246), C23 (H-6, sc-55486) and rabbit polyclonal antibody against human Bax (N-20, sc-493) were from Santa Cruz Biotechnology. Polyclonal antibody against the 1-89 aa region of Capn3b was raised in rabbit by HuaAn Biotechnology (Hangzhou, China).

\section{WISH, cryo-sectioning and immunostaining}

Probe labeling and WISH were performed as described previously [13]. The 3'-UTRs of capn $3 a$ and capn $3 b$ were cloned (Supplementary information, Table S5) and used as probes.

Cryosectioning and immunofluorescence staining were performed as described previously [17]. For immunofluorescence staining of the cultured cells, cells were plated on coverslips in a six-well plate. Primary antibodies were used at a 1:100 dilution. Immunofluorescence was detected after staining with Alexa Fluor conjugated secondary antibody (Invitrogen) (1:400 dilution). DAPI was used to stain the nuclei. All immunofluorescence staining images were taken under a Leica TCS SP5 confocal microscope.

\section{Coimmunoprecipitation (Co-IP)}

Human cell lines transfected with various plasmids were harvested with Tris- $\mathrm{HCl}$ protein lysis buffer for total protein extraction. Co-IP was performed using an HA Tag IP/Co-IP Kit (Pierce) according to the manufacturer's instructions. The input and precipitated protein samples were subjected to western blot analysis with corresponding antibodies as stated.

\section{TUNEL assay and embryo viability counting}

The TUNEL assay was performed using the in situ Cell Death Detection Kit, TMR red (Roche) as described previously [13]. For viability counting, dead embryos were characterized by the presence of large, dark debris in the embryos.

\section{Flow cytometry analysis (FACS)}

Human cells were digested with trypsin and precipitated at $500 \times g, 4^{\circ} \mathrm{C}$. After washing in PBS, the cells were stained with PI (Beyotime) or costained with Annexin V-FITC and PI according to the manufacturer's instructions, and subjected to FACS analysis of the cell cycle and apoptosis using a Beckman Coulter FC 500 $\mathrm{MCL}$ and a BD FACSCalibur flow cytometer, respectively.

\section{Acknowledgments}

We thank Prof Tuanlao Wang (Xiameng University) for providing the MCF-7-1 cell strain, Prof Zhengping Xu (Zhejiang University) for the MCF-7-2 cell strain and HepG2 cell line, Prof Xing Xie (Zhejiang University) for the SiHa cell line, and Prof Cong Liu (Sichuan University) for the OVCAR-8/TR cell line. We thank Yun Feng, Qian Lang, Xin Ye and Menglian Zhang (Zhejiang University) for their help in maintaining the zebrafish facility. This work is financially supported by the National Natural Science Foundation of China (30825025) and National Basic Research Program of China (2012CB944500).

\section{References}

1 Levine AJ, Hu W, Feng Z. The P53 pathway: what questions remain to be explored? Cell Death Differ 2006; 13:1027-1036.

2 Tai E, Benchimol S. TRIMming p53 for ubiquitination. Proc Natl Acad Sci USA 2009; 106:11431-11432.

3 Tsvetkov P, Reuven N, Shaul Y. Ubiquitin-independent p53 proteasomal degradation. Cell Death Differ 2010; 17:103-108.

4 Pariat M, Carillo S, Molinari M, et al. Proteolysis by calpains: a possible contribution to degradation of p53. Mol Cell Biol 1997; 17:2806-2815.

5 Kubbutat MH, Vousden KH. Proteolytic cleavage of human p53 by calpain: a potential regulator of protein stability. Mol Cell Biol 1997; 17:460-468.

6 Gonen H, Shkedy D, Barnoy S, Kosower NS, Ciechanover A. On the involvement of calpains in the degradation of the tumor suppressor protein p53. FEBS Lett 1997; 406:17-22.

7 Qin Q, Liao G, Baudry M and Bi X. Role of calpain-mediated p53 truncation in semaphorin 3A-induced axonal growth regulation. Proc Natl Acad Sci USA 2012; 107:13883-13887.

8 Weber JD, Taylor LJ, Roussel MF, Sherr CJ, Bar-Sagi D. Nucleolar Arf sequesters Mdm2 and activates p53. Nat Cell Biol 1999; 1:20-26.

9 Bernardi R, Scaglioni PP, Bergmann S, Horn HF, Vousden $\mathrm{KH}$, Pandolfi PP. PML regulates p53 stability by sequestering Mdm2 to the nucleolus. Nat Cell Biol 2004; 6:665-672.

10 Dai MS, Lu H. Inhibition of MDM2-mediated p53 ubiquitination and degradation by ribosomal protein L5. J Biol Chem 2004; 279:44475-44482.

11 Lohrum MAE, Ludwig RL, Kubbutat MHG, Hanlon M, Vousden KH. Regulation of HDM2 activity by the ribosomal protein L11. Cancer Cell 2003; 3:577-587.

12 Fumagalli S, Ivanenkov VV, Teng T, Thomas G. Suprainduction of $\mathrm{p} 53$ by disruption of $40 \mathrm{~S}$ and $60 \mathrm{~S}$ ribosome biogenesis leads to the activation of a novel G2/M checkpoint. Genes Dev 2012; 26:1028-1040.

13 Chen J, Ruan H, Ng SM, et al. Loss of function of def selectively up-regulates Delta113p53 expression to arrest expansion growth of digestive organs in zebrafish. Genes Dev 2005; 19:2900-2911.

14 Charette JM, Baserga SJ. The DEAD-box RNA helicase-like Utp25 is an SSU processome component. RNA 2010; 16:21562169. 
15 Goldfeder MB, Oliveira CC. Utp25p, a nucleolar Saccharomyces cerevisiae protein, interacts with U3 snoRNP subunits and affects processing of the 35 S pre-rRNA. FEBS J 2010; 277:2838-2852.

16 Harscoet E, Dubreucq B, Palauqui JC, Lepiniec L. NOF1 encodes an Arabidopsis protein involved in the control of rRNA expression. PLoS One 2010; 5:e12829.

17 Chen J, Ng SM, Chang C, et al. p53 isoform delta113p53 is a p53 target gene that antagonizes p53 apoptotic activity via BclxL activation in zebrafish. Genes Dev 2009; 23:278-290.

18 Bourdon JC, Fernandes K, Murray-Zmijewski F, et al. p53 isoforms can regulate p53 transcriptional activity. Genes Dev 2005; 19:2122-2137.

19 Aoubala M, Murray-Zmijewski F, Khoury MP, et al. p53 directly transactivates Delta133p53alpha, regulating cell fate outcome in response to DNA damage. Cell Death Differ 2011; 18:248-258.

20 Boldrup L, Bourdon JC, Coates PJ, Sjostrom B, Nylander K. Expression of p53 isoforms in squamous cell carcinoma of the head and neck. Eur J Cancer 2007; 43:617-623.

21 Ebrahimi M, Boldrup L, Coates PJ, Wahlin YB, Bourdon JC, Nylander K. Expression of novel p53 isoforms in oral lichen planus. Oral Oncol 2008; 44:156-161.

22 Libault M, Tessadori F, Germann S, Snijder B, Fransz P, Gaudin V. The Arabidopsis LHP1 protein is a component of euchromatin. Planta 2005; 222:910-925.

23 Boisvert FM, van KS, Navascues J, Lamond AI. The multifunctional nucleolus. Nat Rev Mol Cell Biol 2007; 8:574-585.

24 Billy E, Wegierski T, Nasr F, Filipowicz W. Rcllp, the yeast protein similar to the RNA 3'-phosphate cyclase, associates with U3 snoRNP and is required for $18 \mathrm{~S}$ rRNA biogenesis. EMBO J 2000; 19:2115-2126.

25 Berghmans S, Murphey RD, Wienholds E, et al. tp53 mutant zebrafish develop malignant peripheral nerve sheath tumors. Proc Natl Acad Sci USA 2005; 102:407-412.

26 Dai $\mathrm{C}, \mathrm{Gu}$ W. p53 post-translational modification: deregulated in tumorigenesis. Trends Mol Med 2010; 16:528-536.

27 Langheinrich U, Hennen E, Stott G, Vacun G. Zebrafish as a model organism for the identification and characterization of drugs and genes affecting p53 signaling. Curr Biol 2002;
12:2023-2028.

28 Bykov VJ, Issaeva N, Shilov A, et al. Restoration of the tumor suppressor function to mutant $\mathrm{p} 53$ by a low-molecular-weight compound. Nat Med 2002; 8:282-288.

29 Malcikova J, Tichy B, Damborsky J, et al. Analysis of the DNA-binding activity of p53 mutants using functional protein microarrays and its relationship to transcriptional activation. Biol Chem 2010; 391:197-205.

30 Sorimachi H, Hata S, Ono Y. Impact of genetic insights into calpain biology. J Biochem 2011; 150:23-37.

31 Moretti D, Del BB, Cosci E, Biagioli M, Miracco C, Maellaro E. Novel variants of muscle calpain 3 identified in human melanoma cells: cisplatin-induced changes in vitro and differential expression in melanocytic lesions. Carcinogenesis 2009; 30:960-967.

32 Sorimachi H, Toyama-Sorimachi N, Saido TC, et al. Musclespecific calpain, p94, is degraded by autolysis immediately after translation, resulting in disappearance from muscle. $J$ Biol Chem 1993; 268:10593-10605.

33 Kramerova I, Kudryashova E, Tidball JG, Spencer MJ. Null mutation of calpain 3 (p94) in mice causes abnormal sarcomere formation in vivo and in vitro. Hum Mol Genet 2004; 13:1373-1388.

34 Tamura K, Dudley J, Nei M, Kumar S. MEGA4: Molecular Evolutionary Genetics Analysis (MEGA) software version 4.0. Mol Biol Evol 2007; 24:1596-1599.

35 O'Connell MP, Fiori JL, Baugher KM, et al. Wnt5A activates the calpain-mediated cleavage of filamin A. J Invest Dermatol 2009; 129:1782-1789.

36 Jang HS, Lal S, Greenwood JA. Calpain 2 is required for glioblastoma cell invasion: regulation of matrix metalloproteinase 2. Neurochem Res 2010; 35:1796-1804.

(Supplementary information is linked to the online version of the paper on the Cell Research website.)

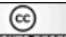

This work is licensed under the Creative Commons Attribution-NonCommercial-No Derivative Works 3.0 Unported License. To view a copy of this license, visit http:// creativecommons.org/licenses/by-nc-nd/3.0 